\title{
Effect of pomegranate (Punica granatum L.) peel powder on the antioxidant and antimicrobial properties of fish gelatin films as active packaging
}

\begin{abstract}
Pomegranate (Punica granatum L.) peel powder was incorporated into fish gelatin film-forming solution (FFS) to develop an active packaging film. The physical, mechanical, antioxidant and antimicrobial properties of the films were investigated. Fish gelatin film without pomegranate peel powder (PPP) was used as the control film. The water vapor permeability (WVP) of the fish gelatin films increased as the content of incorporated PPP increased. However, films with higher PPP contents exhibited higher tensile strengths (TS values), which ranged from 7.48 to 8.02 MPa. PPP significantly $(\mathrm{p}<0.05)$ improved the antioxidant properties of the films in both DPPH and ABTS radical-scavenging activity tests. The film's antimicrobial activities also increased significantly $(\mathrm{p}<0.05)$ after the incorporation of PPP. Staphylococcus aureus $(\mathrm{S}$. aureus) was found to be the most sensitive bacterium to the active film, followed by Listeria monocytogenes (L.monocytogenes) and Escherichia coli (E. coli). The largest inhibition zone $(7.00 \mathrm{~mm})$ was observed for S. aureus around the film incorporated with $5 \%(\mathrm{w} / \mathrm{w}) \mathrm{PPP}$. These results revealed that fish gelatin containing PPP has great potential as an active film with antioxidant and antimicrobial properties, and thus it can help maintain the quality and prolong the shelf life of food products.
\end{abstract}

Keyword: Active packaging; Fish gelatin; Pomegranate peel; Punica granatumL.; Biodegradable polymer 